\title{
Seroprevalence of Mycoplasma pneumoniae in HIV-infected patients using a microparticle agglutination test
}

\author{
Esaki Muthu Shankar, ${ }^{1}$ Nagalingeswaran Kumarasamy, ${ }^{2}$ \\ Pachamuthu Balakrishnan, ${ }^{2}$ A. Vengatesan, ${ }^{3}$ Hayath Kownhar, ${ }^{1}$ \\ Suniti Solomon ${ }^{2}$ and Usha Anand Rao ${ }^{1}$ \\ 1,2Bacteriology Laboratory of the Departments of Microbiology ${ }^{1}$, Dr ALM PG Institute of Basic \\ Medical Sciences, and YRG CARE², VHS, University of Madras, Taramani, Chennai 60011 , \\ India \\ ${ }^{3}$ Clinical Epidemiology Unit, Government Stanley Medical College and Hospital, Chennai \\ 600 113, India
}

Correspondence

Usha Anand Rao

drushaanand@yahoo.com

Received 5 November 2005

Accepted 15 February 2006

\begin{abstract}
Mycoplasma pneumoniae is increasingly recognized as a common and important pathogen in community settings, and is responsible for various pulmonary and extrapulmonary conditions in the normal population. However, the seroepidemiology of acute M. pneumoniae infection in HIV-infected individuals is still unclear worldwide. This study examined the seroprevalence of antibodies to $M$. pneumoniae in HIV-infected patients admitted with respiratory complaints at a tertiary AIDS care centre in Chennai, India. A commercial gelatin microparticle agglutination test (Serodia-Myco II, Fujirebio) was used for the determination of antibodies against M. pneumoniae in acute serum specimens. Of the $200 \mathrm{HIV}$-infected patients with underlying pulmonary conditions tested, 34 (17\% positivity; $95 \% \mathrm{Cl} 12-23 \%$ ) had antibodies specific to M. pneumoniae, while among the 40 patients with no underlying pulmonary symptoms, five (12.5\% positivity; $95 \% \mathrm{Cl} 4-27 \%$ ) had evidence of anti-M. pneumoniae antibody. This shows that the incidence of $M$. pneumoniae seropositivity is greater in patients with underlying pulmonary complaints. Most positive titres were found in the age group 28-37 years in the symptomatic and symptom-free groups ( 64.7 and $60 \%$, respectively). The positive titres ranged from 40 to $>20480$. High titres $(\geqslant 320)$ were found in 10 out of the 39 patients $(25 \cdot 6 \%)$. This seroprevalence study reports a $16 \cdot 2 \%$ prevalence of $M$. pneumoniae infections in HIV-infected patients by a particle agglutination test.
\end{abstract}

\section{INTRODUCTION}

Mycoplasma pneumoniae is an important cause of upper and lower respiratory tract infections, including pharyngitis, tracheobronchitis and pneumonia, in children and adults of all ages (Cassell et al., 1981; Maniloff et al., 1992). Laboratory diagnosis of $M$. pneumoniae infection has relied mainly on serologic tests, because the organism is difficult to isolate (Chamberlein et al., 1983; Cimolai et al., 1996; Fedorko et al., 1995; Karppelin et al., 1993; Tully et al., 1979). A reliable and sensitive serologic test is needed for use in the early phase of infection by M. pneumoniae to confirm the infection and to ensure that the appropriate antibiotic is used for treatment. The detection of specific IgM, which appears 7-10 days after infection and approximately 2 weeks before IgG, has been shown to indicate a recent or current M. pneumoniae infection (Jacobs, 1993; Matas

Abbreviation: $\mathrm{Cl}$, confidence interval. et al., 1998; Sherman et al., 1993; Sillis, 1990). However, the presence of IgM in adult serum does not always indicate a current infection, because in some cases IgM has been shown to persist for up to a year after infection. In addition, the IgM response is minimal or undetectable in some cases of adult reinfection with M. pneumoniae (Chamberlein et al., 1983; Jacobs, 1993; Sherman et al., 1993; Vikerfors et al., 1988). Therefore, reliance on the detection of specific IgM alone, especially in an adult population, could allow some infections to be missed. In a previous study (Vikerfors et al., 1988), approximately $20 \%$ of adults did not mount an IgM response after infection with $M$. pneumoniae.

The seroepidemiology of acute $M$. pneumoniae infection in HIV-infected individuals is still unclear worldwide. Therefore, we tested serum specimens from HIV-infected adult patients with underlying respiratory complaints using the Serodia-Myco II gelatin particle agglutination test for detecting antibodies against $M$. pneumoniae. This test is 
based on the principle that sensitized particles are agglutinated by the presence of antibodies to M. pneumoniae in human serum.

\section{METHODS}

Patients. Sera from $200 \mathrm{HIV}$-infected patients admitted to the inpatient department of YRG-CARE, Chennai, Southern India, with underlying respiratory complaints and $40 \mathrm{HIV}$-infected patients without any pulmonary symptoms during a 1-year period (December 2004 to November 2005) were examined for M. pneumoniae antibody. The study was carried out by protocols approved by the Institutional Review Board (IRB) of YRG CARE, University of Madras, for ethical issues. Written, informed consent was obtained from all patients or their legal representatives before study enrolment. The study was carried out in compliance with good clinical practice, including the International Conference on Harmonization Guidelines and the Declaration of Helsinki.

This was a prospective cohort study, in which the patients were initially screened for HIV infection using HIV double ELISA (HIV-1 and -2), and later confirmed using Western blot assay (positive for HIV-1 antibody) (Immunetics) against gp160 env, gp120 env, p66 pol, p55 gag, gp41 env, p24 gag and p17 gag. Patients that underwent a clinical and radiological diagnosis of respiratory disease and who had new infiltrates on chest X-ray or consolidation which could not be attributed to some other aetiology, in addition to the following symptoms: cough, malaise, fever, sore throat with signs of sputum, dullness, rales/ wheezes or evidence of pulmonary consolidation and myalgia, were considered. The body temperature, pulse rate, blood pressure and $\mathrm{SPO}_{2}$ were recorded from all the patients upon admission. Patients with evidence of nosocomial pneumonia, lung cancer, aspiration pneumonia or bronchiectasis, and those terminally ill were excluded from the study.

Serologic testing. For serologic testing, a single acute blood specimen $(3-5 \mathrm{ml})$ was collected aseptically from all the patients. The sera were separated and stored at $-20{ }^{\circ} \mathrm{C}$ until tested. Prior to testing, samples were thawed and then centrifuged at $3000 \mathrm{~g}$ for $10 \mathrm{~min}$.

Serodia-Myco II is an in vitro diagnostic test for the detection of antibodies to M. pneumoniae which is manufactured using artificial gelatin particles sensitized with cell-membrane components of $M$. pneumoniae (Mac strain). It is based on the principle that the sensitized gelatin particles are agglutinated in the presence of antibodies against M. pneumoniae. Briefly, $25 \mu \mathrm{l}$ of serum was diluted twofold to give dilutions of 1 in 10 to 1 in 10240 . Sensitized and unsensitized particles were added to the 1 in 10 serum dilution to give a final dilution of 1 in 20 . Drops $(25 \mu \mathrm{l})$ of the sensitized particle suspension were then added to the remaining wells, giving final dilutions of 1 in 40 to 1 in 20480 . The plates were shaken for $30 \mathrm{~s}$ and then covered and left undisturbed on a level surface at room temperature for 3 h (Ikeda \& Omori, 2004; Srifuengfung et al., 2004).

The test was initially calibrated using the control serum dilution series. Each batch of tests included control wells containing $25 \mu \mathrm{l}$ of diluent, $25 \mu$ of the particle suspensions and dilutions of a reactive control serum of known titre, supplied with the commercial kit.

Buttons or compact, smooth rings of particles in the bottom of the wells were read as negative agglutination patterns, and a more extensive ring as positive. Titres $\geqslant 40$ were regarded as positive for $M$. pneumoniae antibody.

Standard statistical procedures were adopted to reveal significant findings.

\section{RESULTS}

Serum specimens from $240 \mathrm{HIV}$-infected patients were screened for the presence of antibodies against M. pneumoniae; 200 patients had underlying respiratory conditions, while 40 patients formed the control group without any pulmonary symptoms. Table 1 shows the results of 240 serum specimens tested by Serodia-Myco II particle agglutination. Of the $200 \mathrm{HIV}$-infected patients with underlying pulmonary conditions tested, 34 (17\% positivity; $95 \%$ CI $12-23 \%$ ) had antibodies specific to M. pneumoniae, while among the 40 patients with no underlying pulmonary symptoms, five ( $12.5 \%$ positivity; $95 \%$ CI $4-27 \%)$ showed evidence of anti-M. pneumoniae antibody. This shows that the incidence of $M$. pneumoniae seropositivity was greater in patients with underlying pulmonary complaints. Table 2 shows that the positive titres ranged from 40 to $>20480$. High titres $(>320)$ were found in 10 of the 39 patients $(25 \cdot 6 \%)$. The highest titre of 20480 was found in two $(5 \cdot 1 \%)$ patients, one with respiratory disease and another with no underlying pulmonary ailments. There was no significant difference between the two groups with respect to seropositivity and $\operatorname{sex}(z=0 \cdot 06, P=0 \cdot 95)$. ( $P>0 \cdot 05$ was considered significant). However, there was a significant difference with regard to sex and seropositivity among those without respiratory complaints $(z=3 \cdot 1, P=0 \cdot 002)$. In the HIV-infected subjects with no respiratory complaints, the incidence of seropositivity was $16 \%(n=4)$ in females and $7 \%(n=1)$ in male subjects. The overall positivity of antibodies specific to M. pneumoniae in the HIV-infected patients tested was $16 \cdot 2 \%$.

\section{DISCUSSION}

AIDS is manifested as a collection of diseases resulting from severe immunosuppression due to HIV infection. AIDS is clinically defined as the stage when an HIV-infected person's CD4 + lymphocyte count falls below 200 per microlitre or the HIV-infected person has any of the AIDSdefining clinical illnesses. Mycoplasmal infection is highly significant clinically in AIDS, even if it is only one example of an opportunistic infection, as opportunistic infections are reportedly the direct cause of death in more than $80 \%$ of patients with AIDS (Blanchard \& Montagnier, 1994).

Although studies to determine the prevalence of M. pneumoniae in various parts of the world have been carried out, there is still a paucity of good epidemiological data on the frequency with which atypical pathogens such as $M$. pneumoniae cause community-acquired pneumonia in India (Shankar et al., 2005). Isolation of M. pneumoniae from atypical bacterial pneumonia is considered to be the gold standard for diagnosis. However, isolation requires $2-3$ weeks, which limits its clinical usefulness. Serology is probably the most frequently used method to diagnose M. pneumoniae infections. Moreover, serologic tests are more sensitive indicators of mycoplasma infection than culture of the organism, because in many instances antibody responses can be detected when the organism cannot 
Table 1. M. pneumoniae-positive serum from HIV-infected patients with and without respiratory complaints of different age groups

The number of patients with a positive serum result is shown.

\begin{tabular}{|lcccccc|}
\hline Patient details & \multicolumn{7}{c|}{ Age (years) } \\
\cline { 2 - 6 } & $\mathbf{1 8 - 2 7}$ & $\mathbf{2 8 - 3 7}$ & $\mathbf{3 8 - 4 7}$ & $\mathbf{4 8 - 5 7}$ & $>\mathbf{5 7}$ & Total \\
\hline With respiratory complaints & 1 & 16 & 6 & 2 & 3 & 28 \\
Male $(n=161)$ & - & 6 & - & - & - & 6 \\
Female $(n=39)$ & 1 & 22 & 6 & 2 & 3 & 34 \\
Total $(n=200)$ & $2 \cdot 9$ & $64 \cdot 7$ & $17 \cdot 6$ & $5 \cdot 8$ & $8 \cdot 8$ & $100 \cdot 0$ \\
Percentage positive & - & 2 & 2 & - & - & 4 \\
Without respiratory complaints & - & 1 & - & - & - & 1 \\
Male $(n=25)$ & - & 3 & 2 & - & - & 5 \\
Female $(n=15)$ & - & $60 \cdot 0$ & $40 \cdot 0$ & - & - & $100 \cdot 0$ \\
Total $(n=40)$ & & & & & & \\
Percentage positive & & & & & & \\
\end{tabular}

be recovered (Srifuengfung et al., 2004). Therefore, current routine methods for laboratory diagnosis of $M$. pneumoniae infection are based primarily on serologic analysis (Srifuengfung et al., 2004; Hammerschlag, 2001). Among the various serological tests available for detecting M. pneumoniae infections, ELISA has been described to be the most sensitive and specific (Marmion et al., 1993; Clyde, 1983; Kleemola et al., 1982). We tested a single serum sample from each patient, as this has been reported to be of epidemiologic value and helps in determining the percentage of a population that has actually been exposed to the organism (Velleca et al., 1980).

In addition, the complement fixation test (CFT), a commonly used test, uses a lipid component of M. pneumoniae that cross-reacts with components of mammalian tissue as well as bacterial glycolipids, giving false-positive results (Kenny et al., 1990; Jacobs et al., 1986; Brunner et al., 1977;
Dussaix et al., 1983). The CFT is also limited by its inability to detect specific antibodies other than IgG (Gendrel et al., 1997). Numerous authors have described serology as the standard laboratory method for the diagnosis of $M$. pneumoniae infections (Sillis, 1990). Therefore, our study used serology to look for the seroprevalence of M. pneumoniae infections among HIV-infected subjects. This study was aimed primarily at determining the seroprevalence rate of M. pneumoniae, rather than diagnosing the aetiological agent in HIV-infected individuals. However, information on the reported cross-reactivity between $M$. pneumoniae and Mycoplasma genitalium was not available for this test, although this is recognized to be a problem, particularly with the CFT.

Gendrel et al. (1997) diagnosed M. pneumoniae infection in $42 \%$ of patients. An Indian study has reported that the prevalence rate of $M$. pneumoniae in the normal population

Table 2. Positive serum from HIV-infected patients of different age groups

\begin{tabular}{|c|c|c|c|c|}
\hline \multirow[t]{2}{*}{ Titre } & \multicolumn{3}{|c|}{ Number of positive serum samples $(n=39)$} & \multirow{2}{*}{$\begin{array}{l}\text { Percentage of positive } \\
\text { serum samples }\end{array}$} \\
\hline & $\begin{array}{c}\text { Male } \\
(n=186)\end{array}$ & $\begin{array}{c}\text { Female } \\
(n=54)\end{array}$ & $\begin{array}{c}\text { Total } \\
(n=240)\end{array}$ & \\
\hline 40 & 8 & 1 & 9 & $23 \cdot 0$ \\
\hline 80 & 9 & 5 & 14 & $35 \cdot 8$ \\
\hline 160 & 5 & 1 & 6 & $15 \cdot 3$ \\
\hline 320 & 4 & - & 4 & $10 \cdot 2$ \\
\hline 640 & - & - & - & - \\
\hline 1280 & 1 & - & 1 & $2 \cdot 5$ \\
\hline 2560 & 1 & - & 1 & $2 \cdot 5$ \\
\hline 5120 & 1 & - & 1 & $2 \cdot 5$ \\
\hline 10240 & 1 & - & 1 & $2 \cdot 5$ \\
\hline 20480 & 2 & - & 2 & $5 \cdot 1$ \\
\hline$>20480$ & - & - & - & - \\
\hline Total & 32 & 7 & 39 & $100 \cdot 0$ \\
\hline
\end{tabular}


is about $35 \%$ (Dey et al., 2000), while others have identified it in only $1.9 \%$ of patients (Mundy et al., 1998). Nevertheless, the role of M. pneumoniae in adults with respect to HIV infection needs to be defined.

According to our criteria, $17 \%$ (95\% CI 12-23\%) of those with underlying pulmonary conditions had antibodies specific to M. pneumoniae, while five $(12.5 \%$; $95 \%$ CI $4-27 \%$ ) of the 40 patients with no underlying pulmonary symptoms had evidence of anti-M. pneumoniae. Clyde (1983) has shown that M. pneumoniae accounts for up to $30 \%$ of all pneumonia cases in the normal population. No significant difference could be established between the two groups with respect to seropositivity and $\operatorname{sex}(z=0 \cdot 06$, $P=0 \cdot 95)$ in the present study. Nevertheless, a significant difference was demonstrated with regard to sex and seropositivity among those without respiratory complaints $(z=3 \cdot 1, P=0 \cdot 002)$; this aspect needs further study. The prevalence of $M$. pneumoniae in HIV-infected individuals seems to contradict reports that $M$. pneumoniae is commonly seen in children and young people rather than adults, as our study was carried out in adult patients with HIV. Evidence of infection was a titre of $1: 40$ or greater. However, the specification for this assay did not state whether it detected IgG or IgM. Moreover, the present finding should not be considered to reflect the exact seroprevalence of antibodies against $M$. pneumoniae, as recent studies have shown that HIV-infected adults may not produce measurable IgG against M. pneumoniae (Twigg et al., 1996). In addition, patients continue to produce detectable levels of IgM occasionally, due to incomplete clearance of the organism from the respiratory tract (Atkinson et al., 2004; Twigg et al., 1996).

In conclusion, antibody specific to $M$. pneumoniae was detected in $16 \cdot 2 \%$ of the HIV-infected patients screened. To the best of our knowledge, this study is the first to report the seroprevalence of M. pneumoniae among HIV-infected patients.

\section{ACKNOWLEDGEMENTS}

The authors thank the Indian Council of Medical Research (ICMR), New Delhi [award no. 3/1/(3) 54-2003 MPD-JRF] for financial assistance. The kind help at the inpatient station of S. P. Martin, Arumugam Geetha, Usha, Dhanalakshmi, Hepsiba Beula, Nalini and Rashmi is gratefully acknowledged.

\section{REFERENCES}

Atkinson, T., Duffy, L. B., Pendley, D., Dai, Y. \& Cassell, G. H. (2004). Reduced IgG antibody to Mycoplasma pneumoniae among subjects with asthma: results of a five-year study. J Allergy Clin Immunol 115, S171.

Blanchard, A. \& Montagnier, L. (1994). AIDS-associated mycoplasmas. Annu Rev Microbiol 48, 687.

Brunner, H., Prescott, B., Greenberg, H., James, W. D., Horswood, R. L. \& Chanock, R. M. (1977). Unexpectedly high frequency of antibody to Mycoplasma pneumoniae in human sera as measured by sensitive techniques. J Infect Dis 135, 524-530.

Cassell, G. H. \& Cole, B. C. (1981). Mycoplasmas as agents of human disease. $N$ Engl J Med 304, 80-89.

Chamberlein, P. \& Saeed, A. A. (1983). A study of the specific IgM antibody response in Mycoplasma pneumoniae in man. J Hyg Camb 90, 207-211.

Cimolai, N. \& Cheong, A. C. H. (1996). An assessment of a new diagnostic indirect enzyme immunoassay for the detection of antiMycoplasma pneumoniae IgM. Am J Clin Pathol 105, 205-209.

Clyde, W. A., Jr (1983). Mycoplasma pneumoniae respiratory disease symposium: summation and significance. Yale J Biol 56, 523-527.

Dey, A. B., Chaudhry, R., Kumar, P., Nisar, N. \& Nagarkar, K. M. (2000). Mycoplasma pneumoniae and community-acquired pneumonia. Natl Med J India 13, 66-70.

Dussaix, E., Slim, A. \& Tournier, P. (1983). Comparison of enzymelinked immunosorbent assay (ELISA) and complement fixation test for detection of Mycoplasma pneumoniae antibodies. J Clin Pathol 36, 228-232.

Fedorko, D. P., Emery, D. D., Franklin, S. M. \& Congdon, D. D. (1995). Evaluation of a rapid enzyme immunoassay system for serologic diagnosis of Mycoplasma pneumoniae infection. Diagn Microbiol Infect Dis 23, 85-88.

Gendrel, D., Raymond, J., Moulin, F., Iniguez, J. L., Ravilly, S., Habib, F., Lebon, P. \& Kalifa, G. (1997). Etiology and response to antibiotic therapy of community-acquired pneumonia in French children. Eur J Clin Microbiol Infect Dis 16, 388-391.

Hammerschlag, M. R. (2001). Mycoplasma pneumoniae infections. Curr Opin Infect Dis 14, 181-186.

Ikeda, A. \& Omori, S. (2004). Experience with Serodia-Myco in indirect hemagglutination tests for antibody against Mycoplasma pneumoniae. Serodia-Myco bibliography 21. Tokyo: Fujirebio Inc.

Jacobs, E. (1993). Serological diagnosis of Mycoplasma pneumoniae infections: a critical review of current procedures. Clin Infect Dis 17, S79-S82.

Jacobs, E., Bennewitz, A. \& Bredt, W. (1986). Reaction pattern of human anti-Mycoplasma pneumoniae antibodies in enzyme-linked immunosorbent assays and immunoblotting. J Clin Microbiol 23, 517-522.

Karppelin, M., Hakkarainen, K., Kleemola, M. \& Miettinen, A. (1993). Comparison of three serological methods for diagnosing Mycoplasma pneumoniae infection. J Clin Pathol 46, 1120-1123.

Kenny, G. E., Kaiser, G. G., Cooney, M. K. \& Foy, H. M. (1990). Diagnosis of Mycoplasma pneumoniae: sensitivity and specifications of serology with lipid antigen and isolation of the organism on soy peptone medium for identification of infections. J Clin Microbiol 28, 2087-2093.

Kleemola, M. \& Kayhty, H. (1982). Increase in titer of antibodies to Mycoplasma pneumoniae in patients with purulent meningitis. J Infect Dis 146, 284-288.

Maniloff, J., McElhancy, R. N., Finch, L. R. \& Baseman, J. B. (editors) (1992). Mycoplasmas: Molecular Biology and Pathogenesis. Washington, DC: American Society for Microbiology.

Marmion, B. P., Williamson, J., Worswick, D. A., Kok, T. W. \& Harris, R. J. (1993). Experience with newer techniques for the laboratory detection of Mycoplasma pneumoniae: Adelaide, 1978-1992. Clin Infect Dis 17, S90-S99.

Matas, L., Dominguez, J., De Ory, F., Garcia, N., Gali, N., Cardona, P. J., Hernandez, A., Rodrigo, C. \& Ausina, V. (1998). Evaluation of Meridian ImmunoCard mycoplasma test for the detection of Mycoplasma pneumoniae-specific IgM in paediatric patients. Scand J Infect Dis 30, 289-293. 
Mundy, L. M., Oldach, D., Auwaerter, P. G., Gaydos, C. A., Moore, R. D., Bartlett, J. G. \& Quinn, T. C. (1998). Implications for macrolide treatment in community-acquired pneumonia. Chest 113, 1201-1206.

Shankar, E. M., Kumarasamy, N., Krishnakumar, R., Balakrishnan, P., Solomon, S. \& Usha, A. R. (2005). Recovery rates of Mycoplasma pneumoniae in induced sputum and throat swab specimens of HIV infected patients at an AIDS care centre in Chennai, South India. In Abstracts of the 157th Meeting of the Society for General Microbiology, Keele University, UK, abstract 18. Reading, UK: Society for General Microbiology.

Sherman, M. J., Cubic, H. A. \& Inglis, J. M. (1993). Mycoplasma pneumoniae infection: early diagnosis by detection of specific IgM by immunofluorescence. Br J Biomed Sci 50, 305-308.

Sillis, M. (1990). The limitations of IgM assays in the serological diagnosis of Mycoplasma pneumoniae infections. J Med Microbiol 33, 253-258.
Srifuengfung, S., Techachaiwiwat, W. \& Dhiraputra, C. (2004). Serological study of Mycoplasma pneumoniae infections. J Med Assoc Thai 87, 935-938.

Tully, J. G., Rose, D. L., Whitcomb, R. F. \& Wenzel, R. P. (1979). Enhanced isolation of Mycoplasma pneumoniae from throat washing with a newly modified culture medium. J Infect Dis 139, 478-482.

Twigg, H. L., 3rd, Spain, B. A., Soliman, D. M., Bowen, L. K., Heidler, K. M. \& Wilkes, D. S. (1996). Impaired IgG production in the lungs of HIV-infected individuals. Cell Immunol 170, 127-133.

Velleca, W. M., Bird, B. R. \& Forrester, F. T. (editors) (1980). Laboratory Diagnosis of Mycoplasma Infections. Atlanta, GA: Centres for Disease Control.

Vikerfors, T., Brodin, G., Grandien, M., Hirschberg, L., Krook, A. \& Petterson, C. A. (1988). Detection of specific IgM antibodies for the diagnosis of Mycoplasma pneumoniae infections: a clinical evaluation. Scand J Infect Dis 20, 601-610. 